\title{
MEAN OF MEDIAN ABSOLUTE DERIVATION TECHNIQUE FOR SPECKLE NOISE VARIANCE ESTIMATION IN COMPUTERISED TOMOGRAPHY IMAGES
}

\author{
M. C. Eze ${ }^{1, *}$, O. N. Iloanusi ${ }^{2}$ and C. C. Osuagwu ${ }^{3}$ \\ 1,2,3 DePARTMENT OF EleCtronic ENGINEERING, UNIVERSITY OF NigERIA, NSUKKA. NIGERIA \\ Email addresses:1 martin.eze@unn.edu.ng, ${ }^{2}$ ogehukwu.iloanusi@unn.edu.ng, ${ }^{3}$ chales.osuagwu@unn.edu.ng
}

\begin{abstract}
The accurate estimation of noise variance in an image is the first important stage in image filtering using adaptive filters. In this paper, a new technique for the estimation of speckle noise present in Computerised Tomography (CT) lung image was developed. The development of mean of median absolute derivation technique based on the estimated mean of speckle noise present in CT images is presented. From the result of the simulations, the new technique gave a reasonably accurate estimate of variance of speckle noise present in CT Images. Ten samples of $85 \times 73$ CT images corrupted by speckle noise level ranging from 10\% to 30\% where used as test images. Also, the new technique gave the lowest average speckle noise variance estimation error of 2.53\% compared to 12.53\% for the Median of Median Absolute Derivative Technique, 18.18\% for the Transfer function technique and 37.14\% for the Mode of Variance Technique. The simulation software used in the paper is Matrix Laboratory (MATLAB2012).
\end{abstract}

\section{Keywords: Speckle Noise, CT Image, Adaptive Filter, Variance Estimation, Statistics}

\section{INTRODUCTION}

A CT image is a medical image captured using Computerised Tomography machine. The predominant noise type present in CT images is speckle noise [1]. Speckle noise is a form of multiplicative noise that occurs due to constructive and destructive interference of wave during capturing. The accurate estimation of variance of noise present in a noisy CT image makes suitable filtering possible [2]. The value of noise variance obtained during estimation determines the extent to which the adaptive filter is tuned to achieve good noise suppression. Adaptive filters use the estimated noise variance as an input to determine the extent to vary filter centre-pixel weight or the window size to ensure better noise suppression [2,3]. The priori knowledge of the type of noise present in a noisy image helps in the accuracy of the estimation [5]. The accuracy of the noise variance estimation technique used to estimate the variance of noise present in a noisy image determines the accuracy with which adaptive filters are tuned for effective noise suppression [6]. For effective performance, modern filters are tuned based on the level of the noise present in the noisy image. The estimation of the variance of multiplicative noise present in a noisy image is a very difficult task due to speckle decorrelation $[4,5]$. When a noiseless image $(\Upsilon(m, n))$ is corrupted by speckle noise $(N(m, n))$, the noisy image $(X(m, n))$ is given by (1) [9].

$$
X(m, n)=Y(m, n) \mathrm{N}(m, n)
$$

The noise present in (1) is converted to additive noise as shown in (2) using logarithm transform [7, 8].

$$
\log _{e} X(m, n)=\log _{e} Y(m, n)+\log _{\mathrm{e}} \mathrm{N}(m, m)
$$

The logarithmic transformation is used to transform speckle noise from multiplicative noise to additive noise. For non-speckle noises, other transform techniques such as Ascombe transform (for transforming Poisson noise to additive noise) and Ascombe-like transform (for transforming a combined additive and Poisson-like noise) exist [11]. The logarithmic transformation is applied to speckle noise so that linear filtering is used to suppress noise in the noisy image. When a noisy image is filtered using linear filter, the expression for the logarithm of approximate speckle noise approximate image edge and residual image surface is computed using (3) where $\mathrm{h}(\mathrm{m}, \mathrm{n})$ is the linear filter kernel.

$$
\begin{aligned}
\log _{e} W(m, n)= & \log _{e} X(m, n)-h(m, n) \\
& * \log _{e} X(m, n)
\end{aligned}
$$


The parameter, $\mathrm{W}(\mathrm{m}, \mathrm{n})$ in (3) is made up of the approximate speckle noise and approximate image edges and residual image surface. The variance of speckle noise present in the noisy image is estimated from (3) using speckle noise variance estimation techniques. For more accurate noise variance estimation, nonlinear filter like median filter is used for filtering because it preserves the edge of the filtered image [12]. When an edge preserving filter is used for filtering, the parameter, $\mathrm{W}(\mathrm{m}, \mathrm{n})$ includes the approximate speckle noise, residual image edge and residual image surface.

In this work, a Mean of Median Absolute Derivation Technique for estimating the variable of speckle noise present in an image is developed. The technique uses the statistical mean of noise present in an image to estimate the variance of noise present in CT images and other images with uniform intensity.

Many researchers have worked on the multiplicative noise variance estimation techniques. In [2], data masking using histogram technique was used. It was shown that the noise variance is the square of the standard deviation from the histogram. In [13], a Median of Median Absolute Derivation technique was developed. Transfer function based technique was developed in [14]. Local mean and local standard deviation of noisy image was used in noise variance estimation in [15]. In [16], a Robust Median Estimator technique was used. Principal component analysis technique was used to estimate the noise variance in [17]. In [18], statistical mode of noise present in an image was used to estimate the noise variance and median filter was used as a pre-estimation filter since it preserves image edge. In [19], the mode of local variance of the noise present in an image was used to estimate the noise.

However, in this paper, a Mean of Median Absolute Derivation technique for noise variance estimation is developed. Both the Mean of Median Absolute Derivation technique and Median of Median Absolute Derivation technique used median filter for filtering. The two techniques differ since the new technique used statistical mean property of noise for variance estimation while Median of Median Absolute Derivation technique used statistical median of noise for noise variance estimation.

\section{DEVELOPMENT OF MEAN OF MEDIAN ABSOLUTE DERIVATION TECHNIQUE}

The noise variance estimation technique proposed in this work is the Mean of Median Absolute Derivation
Technique. The proposed technique utilizes the value of noise mean to estimate the speckle noise variance. Noise mean property is selected in this paper because it has established quadratic relationship with noise variance. However, the estimation of speckle noise variance is complicated since some pixels give output that are non-real numbers during initial processing stages of the noise variance estimation. This is because the pixels with zero values gives $-\infty$ after logarithm transformation and gives Not-a-Number $(\mathrm{NaN})$ output when the operation in (3) is carried out. $\mathrm{NaN}$ is a numeric data type representing an undefined value. For that reason, images corrupted by speckle noise is corrected by adding the correction factor $(\beta)$ to the corrupted image $(\mathrm{X}(\mathrm{m}, \mathrm{n}))$ before transformation and (1) is modified as shown in (4).

$$
\begin{aligned}
\log _{e}(X(m, n)+\beta) & \\
& =\log _{e}(Y(m, n) \mathrm{N}(m, n)+\beta)
\end{aligned}
$$

The parameter, $\beta$ is a real number such that $\beta \leq 10^{-7}$. The value of $\beta$ is made very negligible to avoid excessive change in pixel values.

For filtering in this paper, Median filter is used because it preserves the edge of the image [12]. The expression in (5) was obtained when the transformed image in (4) was filtered using median filter and subtracted from (4) which is a modification of work done by [13]. The parameter, $\log _{\mathrm{e}} W(\mathrm{~m}, \mathrm{n})$ is the logarithm of combined approximate speckle noise present in the noisy image, residual image edge and residual image surface due to error during filtering.

$$
\begin{aligned}
\log _{e} W(m, n)= & \log _{e}(X(m, n)+\beta) \\
& -h_{M E D}\left(\log _{e}(X(m, n)+\beta)\right)
\end{aligned}
$$

The parameter $h_{M E D}$ is the median filter function. Convolution operation was omitted because the filtering in (5) is nonlinear filtering not linear filtering. The mean of the right hand side of (5) is obtained as given in (6) where $\mathrm{M}$ and $\mathrm{N}$ are the row and column length of the image respectively.

$$
\text { mean }=\frac{1}{M N} \sum_{m=1}^{M} \sum_{n=1}^{N} \operatorname{abs}\left(\log _{e} W(m, n)\right)
$$

The Algorithm for Noise variance $\left(\delta_{n}^{2}\right)$ estimation technique is given in (7). The expression in (7) is obtained by modeling the relationship between the actual variance $\left(\sigma_{n}^{2}\right)$ of speckle noise present in CT images and estimated noise mean computed in (6) using Quadratic Least Squares Regression Method. The Quadratic Least Squares Regression was used in this modeling because the relationship between variance and mean is quadratic relationship.

$$
\delta_{n}^{2}=0.3907(\text { mean }+1.3570)(\text { mean }-0.1133)
$$


The algorithm in (7) is obtained by plotting a scatter plot of the actual variance against the estimated mean for many CT images for noise variance ranging from $0 \%$ to $40 \%$ and drawing a quadratic curve of best fit. The expressing for the line of best fit is the algorithm in (7). The new technique works for other images but gives unique result when applied to CT images. The mean is chosen in this paper because it best represents the noise variance in the image. Based on observation during simulation, the estimation technique developed in this work is effective for speckle noise variance ranging from $10 \%(0.1)$ to $30 \%$ (0.3).

\section{PERFORMANCE METRICS}

The performance of an estimation technique depends on the performance of the preprocessing filter and the statistical quantity used. To effectively discuss the new technique, the Peak Signal to Noise Ratio (PSNR) and Mean Square Error (MSE) metrics of the preprocessing filter and the estimation error $(\xi)$ of the developed technique are discussed.

\subsection{PSNR and MSE Metrics}

The PSNR of an image is the ratio of the maximum power of the image to the maximum power of the noise distorting the image [20]. For a normalized image, the PSNR can be rewritten as shown in (8). A normalized image is an image in which each pixel value has been divided by 255 .

$$
P S N R=10 \log _{10}\left(\frac{255^{2}}{M S E}\right)
$$

The PSNR is measured in decibel. The PSNR Gain is the PSNR of filtered image minus the PSNR of noisy image. On the other hand, MSE is the average of the squared intensity differences between the filtered image pixels and noiseless image.

$$
M S E=\frac{1}{M N} \sum_{m=1}^{M} \sum_{n=1}^{N}[\mathrm{Y}(m, n)-\bar{Y}(m, n)]^{2}
$$

The mean square error according to [21] is calculated using (9) where $\mathrm{Y}(\mathrm{m}, \mathrm{n})$ is the normalized noiseless image and $\bar{Y}(\mathrm{~m}, \mathrm{n})$ is the filtered normalized image.

\subsection{Noise Estimation Metrics}

The performances of the noise variance estimation techniques are quantified using estimation error $(\xi)$ as shown in (10) [14].

$$
\xi=\frac{a b s\left(\sigma_{n}-\delta_{n}\right)}{\sigma_{n}} \times 100
$$

The parameter, $\sigma_{n}$ is the standard deviation of the actual speckle noise present in the noisy image while $\delta_{n}$ is the estimated standard deviation of speckle noise present in the same image.

\section{STEPS IN USING THE MODEL}

The steps involved in the use of the proposed method in estimating that variance of speckle noise present in a noisy image are as follows:

1. Convert the noisy image to grayscale image and divide each pixel value by 255 to normalize the image.

2. Transform the normalized noisy image using logarithmic transformation.

3. Filter the transformed noisy image using a median filter.

4. Subtract the filtered image from the noisy image to leave approximate noise, residual image edge and residual image surfaces.

5. Compute the absolute values of the output in step 4.

6. Compute the mean of the output of step 5 .

7. Compute the speckle noise variance by substituting the value of mean obtained in step 6 in (7).

\section{RESULTS AND DISCUSSION}

The performance of the developed technique in estimating the variance of speckle noise in images was compared with other estimation techniques using ten samples of $85 \times 73$ CT lung images corrupted by speckle noise of variance ranging from $10 \%(0.1)$ to $30 \%(0.3)$. Noise variance has no unit and is often measured in percentage (\%) or fraction of total noisy image variance. The average estimated speckle noise variances obtained in the analysis using four techniques are shown in Table 1. The average speckle noise variance estimation errors calculated from Table 1 using (10) for the four techniques are as shown in Table 3.

The estimated speckle noise variances using the four techniques are shown in Table 1. From this table, it is seen that the noise variance estimated using the proposed technique is closest to the actual noise variance present in the image for all noise levels compared to other techniques. Also, it can be observed that the estimated noise for the proposed technique becomes more accurate as the actual noise variance present increases.

Based on Table 1, it is concluded that the proposed technique has best average speckle noise variance 
estimation performance for all the noise variance range considered.

The large gap between the performance of the proposed technique and the performances of other techniques is because the proposed technique is developed specially for CT images. CT images were used during the development of the technique to accommodate the inherent properties of images captured using CT machines such as high resolution and quantum noise properties [22].

Table 1: Average estimated speckle noise variance for the four noise estimation techniques

\begin{tabular}{|l|l|l|l|l|l|}
\hline \multirow{2}{*}{ Noise Variance Estimation Technique } & \multicolumn{5}{|c|}{ Actual Noise Variance $\left(\sigma_{n}^{2}\right)$} \\
\cline { 2 - 6 } & 0.10 & 0.15 & 0.20 & 0.25 & 0.30 \\
\cline { 2 - 6 } & \multicolumn{5}{|c|}{ Average Estimated noise variance $\left(\delta_{n}^{2}\right)$} \\
\hline Proposed Technique & 0.0894 & 0.1457 & 0.1902 & 0.2412 & 0.3091 \\
\hline Mode of Local Variance [19] & 0.0322 & 0.0537 & 0.0740 & 0.1133 & 0.1453 \\
\hline Transfer function based technique [14] & 0.1070 & 0.1146 & 0.1248 & 0.1366 & 0.1565 \\
\hline Median of Median Absolute Derivative Technique [13] & 0.0828 & 0.1182 & 0.1471 & 0.1892 & 0.2160 \\
\hline
\end{tabular}

Table 2: Estimated speckle noise variance for proposed and Median of Median Absolute Derivative Technique for different images

\begin{tabular}{|c|c|c|c|c|c|c|}
\hline \multirow[t]{3}{*}{ Noise Variance Estimation Technique } & \multirow[t]{3}{*}{ Image } & \multicolumn{5}{|c|}{ Actual Noise Variance $\left(\sigma_{n}^{2}\right)$} \\
\hline & & 0.10 & 0.15 & 0.20 & 0.25 & 0.30 \\
\hline & & \multicolumn{5}{|c|}{ Average Estimated noise variance $\left(\delta_{n}^{2}\right)$} \\
\hline \multirow[t]{3}{*}{ Proposed Technique } & Lena & 0.0950 & 0.1498 & 0.2009 & 0.2571 & 0.3085 \\
\hline & Pepper & 0.0964 & 0.1445 & 0.1901 & 0.2414 & 0.3112 \\
\hline & Cameraman & 0.2112 & 0.2605 & 0.3030 & 0.3654 & 0.4597 \\
\hline \multirow{3}{*}{$\begin{array}{lcc}\text { Median of Median } & \text { Absolute } \\
\text { Derivative } & \text { Technique [13] } & \end{array}$} & Lena & 0.1008 & 0.1686 & 0.2118 & 0.2467 & 0.2976 \\
\hline & Pepper & 0.0998 & 0.2536 & 0.2018 & 0.2323 & 0.2880 \\
\hline & Cameraman & 0.1308 & 0.1803 & 0.2052 & 0.2998 & 0.3767 \\
\hline
\end{tabular}

Table 3: Average speckle noise estimation error for the four noise estimation techniques

\begin{tabular}{|c|c|c|c|c|c|c|}
\hline \multirow{3}{*}{$\begin{array}{c}\text { Noise Variance Estimation } \\
\text { Technique }\end{array}$} & \multicolumn{5}{|c|}{ Actual Noise Variance $\left(\sigma_{n}^{2}\right)$} & \multirow{3}{*}{$\begin{array}{l}\text { Mean of } \\
\text { Average } \\
\text { Variance } \\
\text { estimation } \\
\text { Error (\%) }\end{array}$} \\
\hline & 0.10 & 0.15 & 0.20 & 0.25 & 0.30 & \\
\hline & \multicolumn{5}{|c|}{ Average Variance Estimation Error (\%) } & \\
\hline Proposed Technique & 5.4537 & 1.4471 & 2.4756 & 1.7839 & 1.5037 & 2.5328 \\
\hline Mode of Local Variance [19] & 43.2550 & 40.1948 & 39.1724 & 32.6828 & 30.3964 & 37.1403 \\
\hline $\begin{array}{l}\text { Transfer function based technique } \\
\text { [14] }\end{array}$ & 3.4360 & 12.5929 & 21.0095 & 26.0919 & 27.7712 & 18.1803 \\
\hline $\begin{array}{l}\text { Median of Median Absolute } \\
\text { Derivative Technique [13] }\end{array}$ & 9.0000 & 11.2457 & 14.2416 & 13.0005 & 15.1570 & 12.5289 \\
\hline
\end{tabular}

The performance of any estimation technique depends on the properties of the image considered. This can be seen in Table 2 when the proposed technique and the Median of Median Absolute derivation Technique are applied to Lena, Cameraman and Pepper images. From Table 2, it is seen that the two techniques give the same result for Lena and Pepper images but the proposed technique gives worse result for Cameraman image.

The difference in performances among the four estimation techniques considered is clearly seen in Table 3. From Table 3, it is observed that the proposed technique has the lowest estimation error showing that it has the best estimation accuracy except for noise variance of 0.10 where
Transfer function technique was lower. This is because Transfer function technique was optimized for estimating noise variance ranging from 0.0 to 0.12 . Looking at Table 3 , it is observed that the estimation error of the proposed technique decreases as the variance of noise present increases for noise variance ranging from 0.10 to 0.15 and from 0.20 to 0.3 but for noise variance ranging from 0.15 to 0.20 the estimation error increases as the noise variance increases.

In this paper, 3X3 Median Filter kernel was used for preprocessing of a noisy image since it causes less blur and preserves the edges of the filtered image. Other simple filters that may be used for the same purpose are Averaging Filter and Binomial Filter (Optimized 
Gaussian Filter). The decision to use Median Filter in this work is justified by considering Table 4 obtained from ten samples of 85X73 CT lung images.

Table 4 shows how the PSNR Gain for the filters varies with the variance of speckle noise present in noisy images. From Table.4, it is observed that Median filter gives the highest average PSNR Gain (3.6591dB) compared to Averaging filter $(2.8479 \mathrm{~dB})$ and Binomial filter (3.0140dB).

From Table 4, it is observed that for all noise levels greater than 15\%, Median filter gives the largest values of average PSNR Gain. However, for all noise levels less that $12 \%$, the Median filter has the least
PSNR Gain among the three filters. The increase in average PSNR Gain with increase in the noise variance for Median filter is because median filter is nonlinear filter and is therefore the most stable filter among the three filters.

This shows that median filter gives the best result and therefore is the best filter for the preprocessing.

\section{CT LUNG IMAGES USED IN THE PAPER}

The CT images used in the paper are shown in Figure 1 . The images are of sizes $85 \times 73$. Figure 2 shows the images when each is corrupted by speckle noise of $30 \%$ total variance.

Table 4: Average PSNR Gain for three preprocessing filters

\begin{tabular}{|c|c|c|c|c|c|c|}
\hline \multirow[t]{3}{*}{ Preprocessing Technique } & \multicolumn{5}{|c|}{ Actual Noise Variance $\left(\sigma_{n}^{2}\right)$} & \multirow{3}{*}{$\begin{array}{l}\text { Mean of Average } \\
\text { PSNR Gain (dB) }\end{array}$} \\
\hline & 0.10 & 0.15 & 0.20 & 0.25 & 0.30 & \\
\hline & \multicolumn{5}{|c|}{ Average PSNR Gain (dB) } & \\
\hline 3X3 Median Filter & 3.0946 & 3.3119 & 3.7511 & 3.9574 & 4.1805 & 3.6591 \\
\hline 3X3 Averaging Filter & 3.2544 & 3.1302 & 3.0490 & 2.6649 & 2.1412 & 2.8479 \\
\hline 3x3 Binomial Filter & 3.6862 & 3.3507 & 3.1576 & 2.7134 & 2.1620 & 3.0140 \\
\hline
\end{tabular}

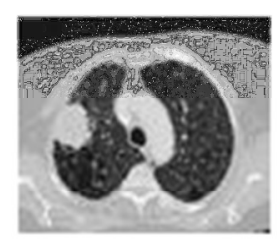

Image 1

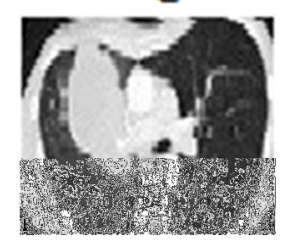

Image6
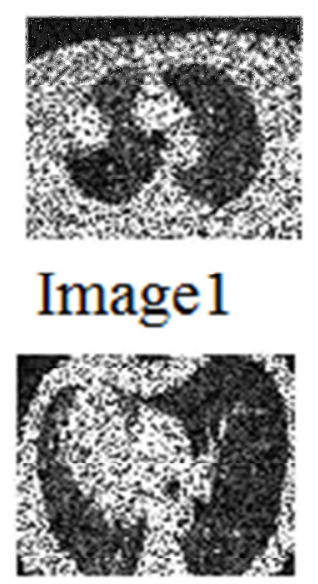

Image6
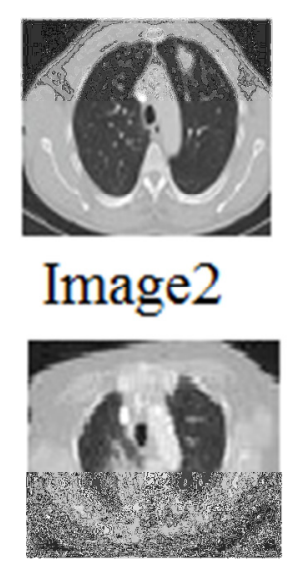

Image 7

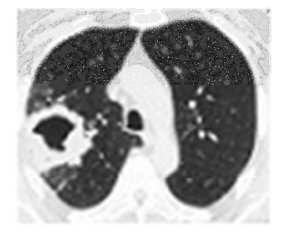

Image 3

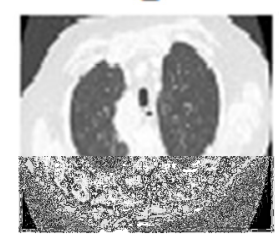

Image 8
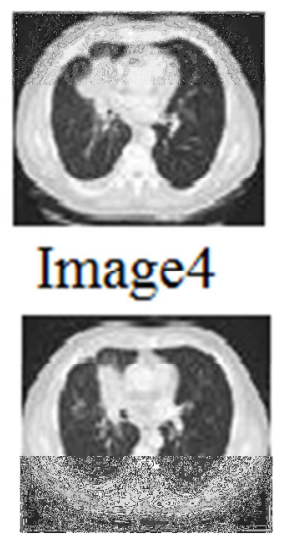

Image9

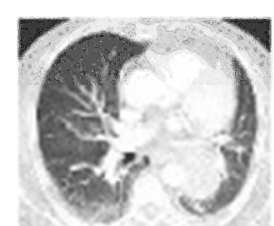

Image 5

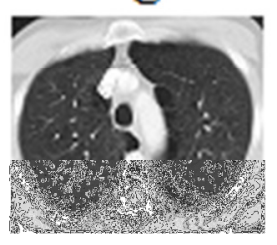

Image 10

Figure 1: Noiseless images used in the paper

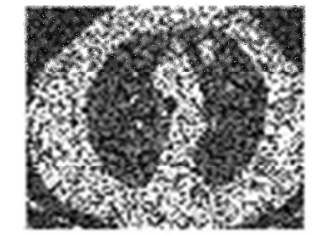

Image 2

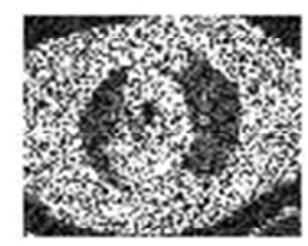

Image 7
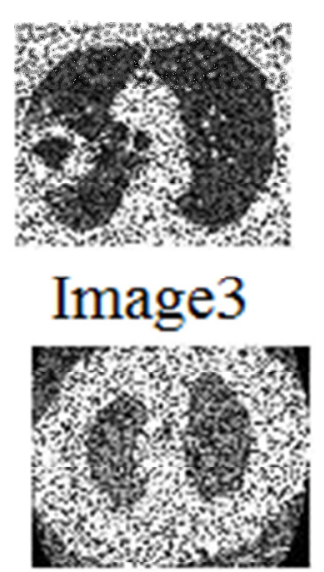

Image 8

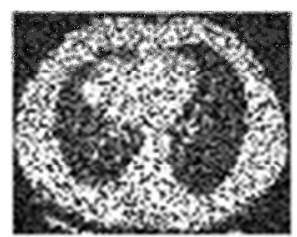

Image 4

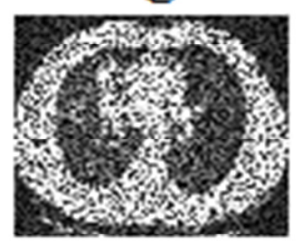

Image9

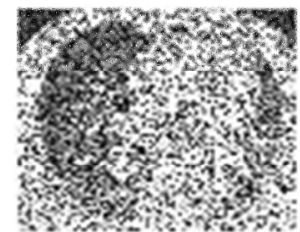

Image 5

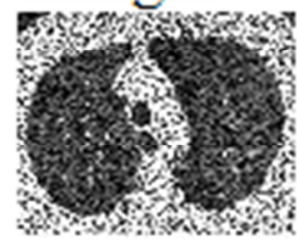

Image 10

Figure 2: Unfiltered images corrupted by speckle noise of $30 \%$ total variance 


\section{CONCLUSION}

In this paper, a speckle noise estimation technique based on the Mean of Median Absolute Derivation was developed. The developed technique estimates the speckle noise variance in a noisy CT image with an average estimation error as low as $2.5328 \%$ for noise variance ranging from $10 \%$ to $30 \%$. The new technique out performs other techniques used to verify its performance including the Median of Median Absolute Derivative Technique which gave an error of $12.5289 \%$. Median filter was selected for the preprocessing before noise estimation since it preserves image edges and performs better than Averaging filter and Binomial filter in suppressing speckle noise, especially at high noise level.

\section{ACKNOWLEDGEMENT}

We thank immensely Conquest Medical Imaging, Trans Ekulu, Enugu for their invaluable technical contribution during this research.

\section{REFERENCES}

[1] Biradar, N., Dewal, M. L. and Rohit, M. "Speckle Noise Reduction using Hybrid TMAV based Fuzzy Filter," International Journal of Research in Engineering and Technology, vol. 3, no. 3, pp. 113-118, 2014.

[2] Corner ,B. R., Narayanan R. M. and Reichenbach, S. E. "Noise estimation in remote sensing imagery using data masking," International Journal of Remote Sensing, vol. 24, no. 4, pp. 697-702, 2003.

[3] Juneja, M. and Mohana , R. "An Improved Adaptive Median Filtering Method for Impulse Noise Detection," International Journal of Recent Trends in Engineering, vol. 1, no. 1, pp. 274-278, 2009.

[4] Eze ,M. C., Iloanusi, O. N., Nnolim , U. A., and Osuagwu , C. C. "Homomorphic Filtering of Speckle Noise From Computerized Tomography ( CT ) Images Using Adaptive Centre-Pixel-Weighed Exponential Filter," International Journal of Image Processing (IJIP), vol. 8, no. 6, pp. 455-467, 2014.

[5] Ganesh, J. S. and Rao, V. S. "Enhanced Noise Type Recognition Using Statistical Measures," IOSR Journal of Computer Engineering, vol. 2, no. 1, pp. 19-23, 2012.

[6] Aja-Fernández, S. and Alberola-López ,C. “On the estimation of the coefficient of variation for anisotropic diffusion speckle filtering.," IEEE transactions on image processing: a publication of the IEEE Signal Processing Society, vol. 15, no. 9, pp. 2694-2701, Sep. 2006.

[7] Lin , C.-H., Sun, Y.-N. and Lin, C.-J. "A motion compounding technique for speckle reduction in ultrasound images.," Journal of digital imaging, vol. 23, no. 3, pp. 246-257, Jun. 2010.

[8] Pregitha, R. E. and Selvakumar, C. E. "Speckle Noise Reduction in Ultrasound Fetal Images Using Edge Preserving Adaptive Shock Filters," International Journal of Scientific and Research Publictions, vol. 2, no. 3, pp. 1-3, 2012.

[9] Padmavathi, G., Subashini, P., Kumar, M. M., and Thakur, S. K. "Comparison of Filters used for Underwater Image Pre-Processing," International Journal of Computer Science and Network Security, vol. 10, no. 1, pp. 58-65, 2010.

[10] Mathur, U., Gill, S. S., and Rattan, M. "Removal of Speckle Noise from Eye Images through Bacterial Foraging Optimization," International Journal of Advanced Engineering Technology, vol. 2, no. 1, pp. 112-119, 2011.

[11] Abramov, S., Zabrodina, V., Lukin, V., Vozel, B., Chehdi, K., and Astola, J. "Methods for Blind Estimation of the Variance of Mixed Noise and Their Performance Analysis," INTECH Numeric Analysis, pp. 49-70, 2011.

[12] Ramamoorthy, K., Chelladurai, T., Sundararajan, P. N., and Krishnamurthy, M. "Noise Suppression using Weighted Median Filter for Improved Edge Analysis in," International Journal of Computer Science and Mobile Computing, vol. 3, no. 1, pp. 97105,2014

[13] Cameron, A., Lui, D., Boroomand, A., Glaister, J., Wong, A., and Bizheva, K. "Stochastic speckle noise compensation in optical coherence tomography using non-stationary spline-based speckle noise modelling.," Biomedical optics express, vol. 4, no. 9, pp. 1769-1785, Jan. 2013.

[14] Kutty, K. and Ojha, S. "A Generic Transfer Function based Technique for Estimating Noise from Images," International Journal of Computer Applications, vol. 51, no. 10, pp. 26-32, 2012.

[15] Aja-Fernández, S., Vegas-Sánchez-Ferrero, G., Martín-Fernández, M., and Alberola-López, C. "Automatic noise estimation in images using local statistics. Additive and multiplicative cases," Image and Vision Computing, vol. 27, no. 6, pp. 756-770, May 2009.

[16] Sudha, S., Suresh, G. R., and Sukanesh, R. "Speckle Noise Reduction in Ultrasound Images by Wavelet Thresholding based on Weighted Variance," International Journal of Computer Theory and Engineering, vol. 1, no. 1, pp. 7-12, 2009.

[17] Pyatykh, S., Hesser, J., and Zheng, L. "Image noise level estimation by principal component analysis.," IEEE transactions on image processing: a publication of the IEEE Signal Processing Society, vol. 22, no. 2, pp. 1-13, Mar. 2013. 
[18] May, N., Alonso, G., and Freire, J. "Robust single image noise estimation from approximate local statistics," in Proceedings of the Twenty-Third Annual Symposium of the Pattern Recognition Association of South Africa, Pretoria South Africa, November 29-30, 2012, pp. 47-53.

[19] Rajan, J., Poot, D., Juntu, J., and Sijbers, J. "Noise measurement from magnitude MRI using local estimates of variance and skewness," Physics in Medicine and Biology, vol. 55, pp. 441-449, 2010.

[20] KlogoGriffith, S., Akpeko, G., and Isaac, A. K. E. "On T he Performance of Filters for Reduction of Speckle Noise in SAR Images of the Coast of the Gulf of Guinea," International Journal of Information Technology, Modeling and Computing, vol. 1, no. 4, pp. 43-52, 2013.

[21] Stella, A. and Trivedi, B. "Implementation of Order Statistic Filters on Digital Image and OCT Image : A Comparative Study," International Journal of Modern Engineering Research, vol. 2, no. 5, pp. 3143-3145, 2012.

[22] Solomon, J. and Samei, E. "Quantum noise properties of CT images with anatomical textured backgrounds across reconstruction algorithms: FBP and SAFIRE," Medical Physics, vol. 41, no. 9, pp. 1-12, 2015. 